\title{
A New Relationship on the Partition Equilibrium of Carboxylic Acids between 1-Octanol/Water and Benzene/Water Systems
}

\author{
Hiromichi Yamada, Tetsuyoshi NaIto, Kazuto Miwa, Noriyuki Tachi and Hiroko Wada \\ Department of Applied Chemistry, Nagoya Institute of Technology, Gokiso, Showa, Nagoya 466, Japan
}

\begin{abstract}
The partition constants of a number of carboxylic acids were determined for the partition systems using 1-octanol and benzene as a solvent, and the dimerization constants of carboxylic acids in the benzene phase were also determined. A linear relationship between the partition constants $\left(\log K_{\mathrm{D}, \mathrm{HA}}\right)$ of carboxylic acids and the molar volume of the respective carboxylic acids was found. A new good relationship on the partition equilibrium of carboxylic acids was found between the partition systems using 1-octanol and benzene, that is, a good linear relationship exists between the values of $\left(\log K_{2, \mathrm{HA}}+2 \log K_{\mathrm{D}, \mathrm{HA}}\right)$ for benzene/water system and the values of $\log K_{\mathrm{D}, \mathrm{HA}}$ for 1-octanol/water system, irrespective of the kinds of carboxylic acids.
\end{abstract}

Keywords Partition equilibrium, carboxylic acid, partition constant, dimerization constant

The additivity of the partition constant of organic compounds was first presented for 203 kinds of monoand disubstituted benzenes by Fujita et al. ${ }^{1}$ In their study the constant $\pi$ was defined as: $\pi_{\mathrm{X}}=\log K_{\mathrm{D}, \mathrm{X}}-\log K_{\mathrm{D}, \mathrm{H}}$, where $K_{\mathrm{D}, \mathrm{X}}$ is a partition constant of a derivative and $K_{\mathrm{D}, \mathrm{H}}$ is that of the parent compound. Subsequently, a series of investigations on the partition constant of organic compounds have been carried out by Hansch et al. ${ }^{2-5}$ The effect of intramolecular hydrophobic bonding on the partition constant of various organic compounds was studied. ${ }^{2}$ The linear free energy relationship between the partition constant and the aqueous solubility of organic liquids was presented. ${ }^{3}$ In addition, the inductive, resonance, steric, branching and conformational effects on the additivity of $\log K_{\mathrm{D}}$ were reviewed., 4

Carboxylic acids are well known to be dimerized in nonsolvating solvents by hydrogen bonding. In order to reveal the partition behavior of a carboxylic acid, it is indispensable to have the information about the dimerization of a carboxylic acid in the organic phase.

The benzene/water and 1-octanol/water systems were selected as a subject of the present investigation in order to make available as many results as possible, since we have many sets of information on the partition equilibrium of various carboxylic acids for the benzene and 1-octanol systems. In the present work, the partition constant of carboxylic acids was found to give linear plots against the molar volume of the respective acids for the 1-octanol/ water system, but the linear plots have proven to vary widely for the benzene/water system. In addition, it has been found that a good linear relationship holds between the values of $\left(\log K_{2, \mathrm{HA}}+2 \log K_{\mathrm{D}, \mathrm{HA}}\right)$ for the benzene/water system and the values of $\log K_{\mathrm{D}, \mathrm{HA}}$ for the 1-octanol/water system, where $K_{2, \mathrm{HA}}$ and $K_{\mathrm{D}, \mathrm{HA}}$ denote the dimerization and partition constants of carboxylic acids. Making use of this linear relationship together with the additivity of the partition constant of organic compounds, the dimerization constant of a carboxylic acid in an inert solvent was estimated. This method is useful for the determination of the dimerization constant of a carboxylic acid, whose partition ratio is difficult to estimate under certain conditions.

\section{Experimentals}

\section{Reagents}

The partition of the following commercial carboxylic acids was carried out at the aqueous ionic strength of $0.1 \mathrm{~mol} \mathrm{dm}^{-3}(\mathrm{H}, \mathrm{Na}) \mathrm{ClO}_{4}$ : 3-phenylpropanoic (T), 2phenylbutanoic (T), 4-phenylbutanoic (T), cyclopentanecarboxylic (A), 2-propenoic (W), trans-2-butenoic (W), 2-hexenoic (A), 3-cyclohexene-1-carboxylic (T) and trans-3-phenyl-2-propenoic (cinnamic, W) acids for both the 1-octanol/water and benzene/water systems, and butanoic (W), pentanoic (W), heptanoic (W) and cyclohexanecarboxylic (N) acids for the former system, and $m$ methylbenzoic acid ( $T$ ) for the latter system, where $T, A$, $\mathrm{W}$ and $\mathrm{N}$ denote Tokyo Kasei Kogyo Co., Ltd., Aldrich Chemical Company, Inc., Wako Pure Chemical Industries, Ltd. and Nacalai Tesque Inc., respectively. 4Phenylbutanoic acid was recrystallized from water. 1Octanol and benzene were purified by the same method as that previously described. ${ }^{6}$ The other carboxylic acids and all other reagents were of reagent grade and were used without further purification. 


\section{Procedure}

A volume of $15 \mathrm{ml}$ each of 0.1 or $0.01(0.001$ for heptanoic acid) mol dm $\mathrm{dm}^{-3}$ perchloric acid aqueous solution kept at $0.1 \mathrm{~mol} \mathrm{dm}^{-3}$ ionic strength and 1octanol and benzene containing the respective carboxylic acids ranging in specified concentrations were mixed in a $50 \mathrm{ml}$ centrifuge tube. Shaking for about $1 \mathrm{~h}$ was carried out in a thermostatic bath kept at $25^{\circ} \mathrm{C}$ and was sufficient for complete equilibration. After centrifuging for $5 \mathrm{~min}$ at $3000 \mathrm{rpm}$, the concentrations of the respective carboxylic acids were determined by the potentiometric titration or by measuring the absorbance at the wavelengths of their absorption maxima. The concentration in the organic phase was calculated by deducting that in the aqueous phase from the total concentration of the carboxylic acid. The dissociation constants of the acids were potentiometrically deter-

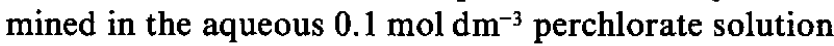
at $25^{\circ} \mathrm{C}$.

\section{Apparatus}

A Cool Bath Shaker ML-10 (TAITEC Co., Koshigaya) kept at $25^{\circ} \mathrm{C}$ was used for mechanical shaking. For centrifugation a Table Top Centrifuge Model 5100 (Kubota Seisakusho Ltd., Tokyo) was used. A DKK ION Meter, Model 10L-50 (DKK Co., Tokyo) was used for the potentiometric titration. Spectrophotometric determination of the carboxylic acids was carried out with a Ubest-55 UV/VIS Spectrophotometer (JASCO, Tokyo).

\section{Results and Discussion}

Determination of the partition and dimerization constants of carboxylic acids

The partition ratio of a carboxylic acid can be expressed as:

$$
D=\frac{C_{\mathrm{HA}, \mathrm{o}}}{C_{\mathrm{HA}, \mathrm{w}}}=\frac{K_{\mathrm{D}, \mathrm{HA}}+2 K_{2, \mathrm{HA}} K_{\mathrm{D}, \mathrm{HA}}{ }^{2}[\mathrm{HA}]}{1+K_{\mathrm{a}} /\left[\mathrm{H}^{+}\right]},
$$

where $K_{\mathrm{D}, \mathrm{HA}}, K_{2, \mathrm{HA}}, K_{\mathrm{a}}$ and [HA] denote the partition constant between the two phases, the dimerization constant in the organic phase, the dissociation constant in the aqueous phase of carboxylic acid, and the concentration of the monomeric carboxylic acid in the aqueous phase, respectively. Under the present partition conditions, the dissociation of the carboxylic acid in the aqueous phase can be neglected, as described in the experimental procedure, that is, $1 \gg K_{\mathrm{a}} /\left[\mathrm{H}^{+}\right]$. Then, the following equation can be obtained from the above one:

$$
\log D=\log K_{\mathrm{D}, \mathrm{HA}}+\log \left(1+2 K_{2, \mathrm{HA}} K_{\mathrm{D}, \mathrm{HA}}[\mathrm{HA}]\right)
$$

If $\log D-\log K_{\mathrm{D}, \mathrm{HA}}=Y$ and $2 K_{2, \mathrm{HA}} K_{\mathrm{D}, \mathrm{HA}}[\mathrm{HA}]=X$, Eq. (2) is equivalent to $Y=\log (1+X)$. According to the above expression, by fitting the plots of $\log D$ against $\log [\mathrm{HA}]$

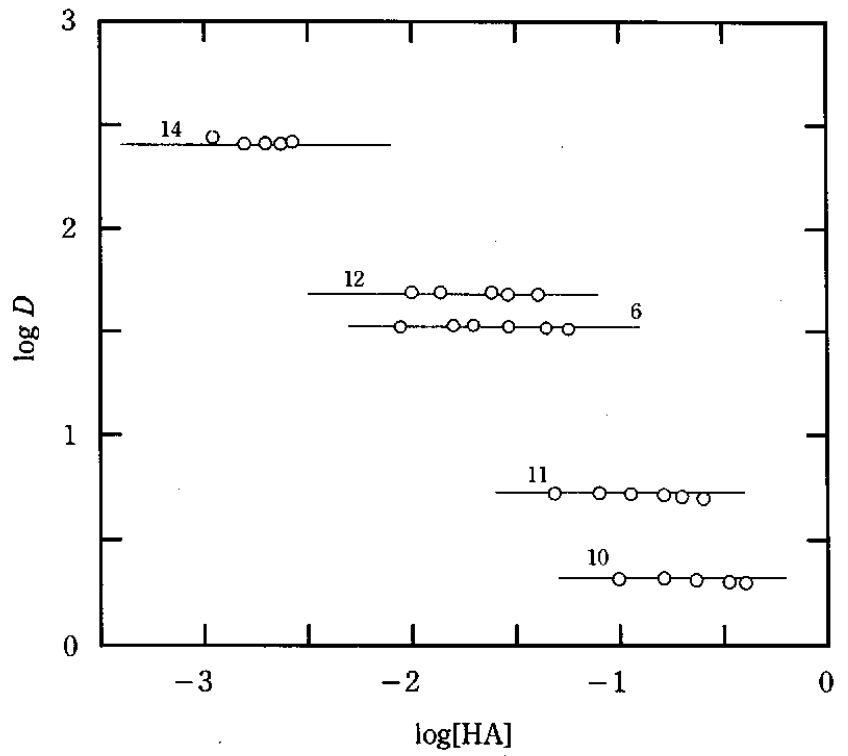

Fig. 1 Determination of the partition constants of carboxylic acids in the 1-octanol/water system. Numbers denote the respective carboxylic acids in Table 1.

with the normalized curve, $\log (1+X) v s . \log X$, the partition and dimerization constants can be estimated from the respective deviations of both the coordinates between the plots and the normalized curve. A few typical plots of $\log D$ against $\log [\mathrm{HA}]$ based on Eq. (2) are depicted for 1-octanol/water system in Fig. 1. The plots for each carboxylic acid are parallel to the horizontal axis. The dimerization of each acid in the 1octanol phase was found not to occur to any appreciable extent as expected. The partition constants of the respective acids can be obtained from the respective distribution ratio for each acid.

On the other hand, the plots of $\log D$ against $\log [\mathrm{HA}]$ are depicted in Fig. 2 for the respective typical systems using benzene as a solvent. The plots fit well with the normalized curve, $\log (1+X)$ vs. $\log X$, as shown in Fig. 2. The partition and dimerization constants of the respective acids were determined from the differences in the ordinates $\left(\log K_{\mathrm{D}, \mathrm{HA}}\right)$ and abscissas $\left(\log 2+\log K_{2, \mathrm{HA}}+\right.$ $\left.\log K_{\mathrm{D}, \mathrm{HA}}\right)$ between the plots and the normalized curve. These values gave good agreement with those obtained by the least-squares method for the plots of $D$ against [HA] on the basis of $D=K_{\mathrm{D}, \mathrm{HA}}+2 K_{2, \mathrm{HA}} K_{\mathrm{D}, \mathrm{HA}^{2}}[\mathrm{HA}$. The values are summarized in Table 1 together with those of the other carboxylic acids including the results presented previously by us.

\section{Effect of molar volume of carboxylic acids on their partition constants}

The additivity of the partition constant of organic compounds is well known and the partition constant in the homologous series is known to increase linearly with the number of the methylene substituents involved in the compounds. The linear correlation of the partition 
Table 1 Partition and dimerization constants of carboxylic acids

\begin{tabular}{|c|c|c|c|c|c|c|c|}
\hline \multirow{2}{*}{\multicolumn{2}{|c|}{ Acid }} & \multirow{2}{*}{$V_{\mathrm{HA}^{\mathrm{a}}}^{\mathrm{a}} / \mathrm{cm}^{3}$} & \multirow{2}{*}{$\mathrm{p} K_{\mathrm{a}}$} & \multirow{2}{*}{$\frac{1-\text { Octanol }}{\log K_{\mathrm{D}, \mathrm{HA}}}$} & \multicolumn{3}{|c|}{ Benzene } \\
\hline & & & & & $\log K_{\mathrm{D}, \mathrm{HA}}$ & $\log K_{2, \mathrm{HA}}$ & $\log K_{2, \mathrm{HA}}+2 \log K_{\mathrm{D}, \mathrm{HA}}$ \\
\hline 1 & Trimethylacetic & $112.9^{7}$ & $4.83^{10}$ & $1.42^{10}$ & $0.02^{10}$ & $2.16^{10}$ & 2.20 \\
\hline 2 & Phenylacetic & $110.9^{7}$ & $4.13^{11}$ & $1.42^{11}$ & $0.19^{11}$ & $1.68^{11}$ & 2.06 \\
\hline 3 & 3-Phenylpropanoic & $127.4^{b}$ & 4.47 & 1.94 & 0.80 & 1.75 & 3.35 \\
\hline 4 & 2-Phenylbutanoic & $143.9^{b}$ & 4.17 & 2.47 & 1.35 & 1.72 & 4.42 \\
\hline 5 & 4-Phenylbutanoic & $143.9^{\mathrm{b}}$ & 4.60 & 2.43 & 1.33 & 1.81 & 4.47 \\
\hline 6 & Cyclopentanecarboxylic & $108.4^{7}$ & 4.78 & 1.52 & 0.07 & 2.33 & 2.47 \\
\hline 7 & Cyclohexanecarboxylic & $124.1^{7}$ & $4.89^{12}$ & 2.01 & $0.90^{12}$ & 1.5412 & 3.34 \\
\hline 8 & Benzoic & $96.5^{7}$ & $4.04^{13}$ & $1.88^{13}$ & $0.14^{6}$ & $2.42^{6}$ & 2.70 \\
\hline 9 & $m$-Methylbenzoic & $129.2^{7}$ & $4.09^{14}$ & $2.41^{14}$ & 0.74 & 2.47 & 3.95 \\
\hline 10 & 2-Propenoic (acrylic) & $68.6^{7}$ & $4.26^{15}$ & 0.31 & -1.09 & 1.48 & -0.70 \\
\hline 11 & trans-2-Butenoic (crotonic) & $84.6^{7}$ & $4.69^{15}$ & 0.71 & -0.28 & 1.00 & 0.44 \\
\hline 12 & 2-Hexenoic & $120.3^{9}$ & $4.75^{16}$ & 1.69 & 1.08 & 0.48 & 2.64 \\
\hline 13 & 3-Cyclohexene-1-carboxylic & $116.7^{9}$ & 4.60 & 1.69 & 0.84 & 0.95 & 2.63 \\
\hline 14 & trans-3-Phenyl-2-propenoic (cinnamic) & $118.8^{7}$ & $4.27^{15}$ & 2.42 & 1.16 & 1.84 & 4.16 \\
\hline
\end{tabular}

a. Molar volume was calculated by $V_{\mathrm{HA}}=$ molecular weight/density cited from the respective references. b. These molar volumes were calculated by adding $16.5 \mathrm{~cm}^{3}$ per one methylene moiety ${ }^{8}$ to the molar volume of phenylacetic acid.

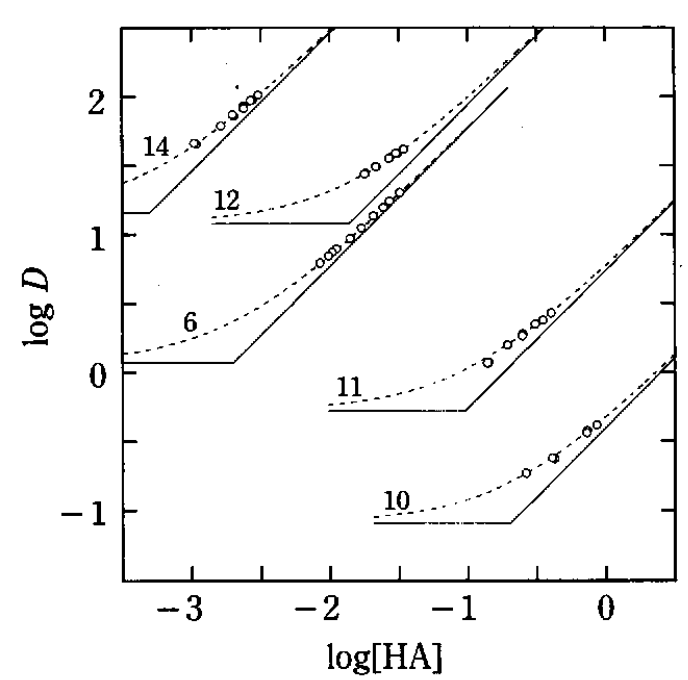

Fig. 2 Determination of the partition and dimerization constants of carboxylic acids in the benzene/water system. The dotted curves and solid lines are the normalized curve, $\log (1+X)$ vs. $\log X$ and the asymptotes of the normalized curve, respectively. Numbers denote the carboxylic acids in Table 1.

constants of a series of the straight-chain aliphatic carboxylic acids from acetic to hexanoic acids with the number of carbon atoms in the acids and with the difference in molar volume between acetic and the respective acids was revealed to be irrespective of the solvents, which are 2-ethylhexyl alcohol, isopropyl ether, nitrobenzene, 1,2-dichloroethane and benzene. ${ }^{17}$

The plots of $\log K_{\mathrm{D}, \mathrm{HA}}$ against the number of carbon atoms involved in the respective carboxylic acids are shown in Fig. 3. As shown in Fig. 3, the plots for both the 1-octanol/water and benzene/water systems can be

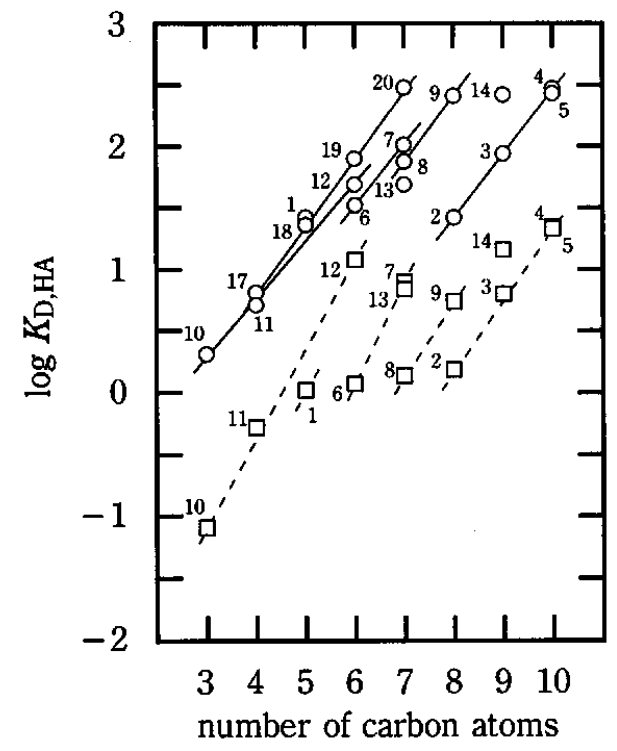

Fig. 3 Correlation of the partition constant of carboxylic acids with the number of carbon atoms involved in them. Symbols $O$ and $\square$ refer to the 1-octanol/water and benzene/ water systems, respectively. Numbers denote the carboxylic acids in Tables 1 and 2.

divided into five groups, which refer to the saturated chain aliphatic, unsaturated aliphatic, aromatic, phenylcarboxylic and cycloalkanecarboxylic acids, with a few exceptions. This suggests that the additivity of the partition constant of a carboxylic acid for the number of carbon atoms in the acid is dependent on the kinds of the carboxylic acids, that is, holds individually for each carboxylic acid group.

The plots of the partition constant of the monomeric carboxylic acids $\left(\log K_{\mathrm{D}, \mathrm{HA}}\right)$ against the molar volume of 


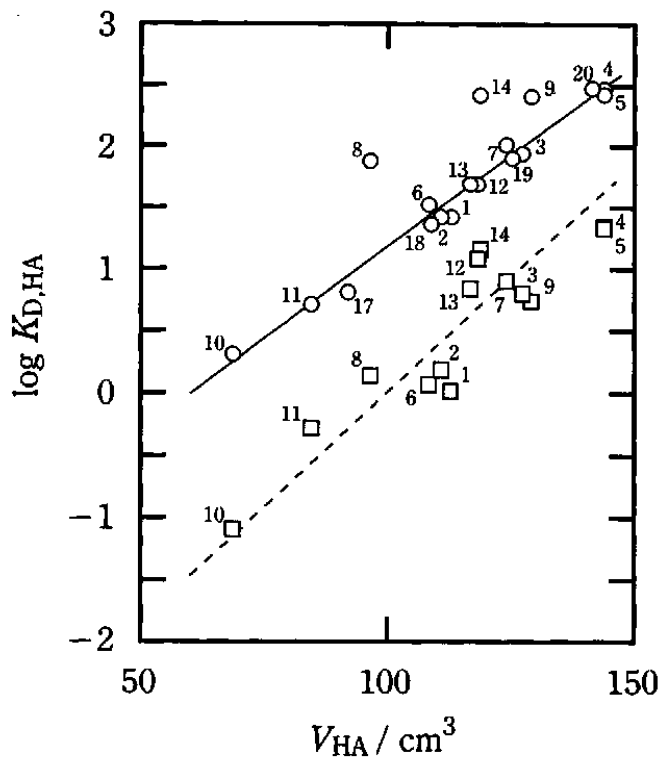

Fig. 4 Relationship between the partition constant of carboxylic acids and their molar volumes. Numbers and symbols are the same as in Fig. 3.

each acid $\left(V_{\mathrm{HA}}\right)$ are depicted in Fig. 4. In contrast to Fig. 3, the plots for the 1-octanol/water system fit with a straight line, with the exception of benzoic (No.8), $m$ methylbenzoic (No.9) and cinnamic (No.14) acids, in which conjugated double bonds exist. The existence of conjugated double bonds can be expected to influence the partition of a carboxylic acid. Although the plots for the cases using benzene as a solvent vary widely, a linear correlation of $\log K_{\mathrm{D}, \mathrm{HA}}$ of the carboxylic acids with each molar volume is roughly observed, with a few exceptions. Judging from these results, the partition constants of a carboxylic acid can be roughly estimated from the molar volume of the respective acids.

\section{Solvent effect on the partition of carboxylic acids}

The plots of the partition constants of the monomeric carboxylic acids for the partition systems using 1-octanol and benzene as an organic phase are shown in Fig. 5. A linear relationship for the partition constants of the carboxylic acids between these systems is observed, though the plots vary widely, as expected from Fig. 4. The partition constant of the monomeric carboxylic acid for the 1-octanol/water system is considerably larger than that for the benzene/water system. The affinity of the monomeric carboxylic acid for 1-octanol is suggested to be stronger than that for benzene. This is due to the solvation of 1-octanol to the monomeric carboxylic acid.

The correlation of the dimerization constant of carboxylic acids with their $p K_{a}$

A linear free energy relationship (LFER) has been observed between the formation of dimeric acids and acid monomer monohydrates and the dissociation of the acids in water for acetic, mono-, di-, and trichloroacetic acids

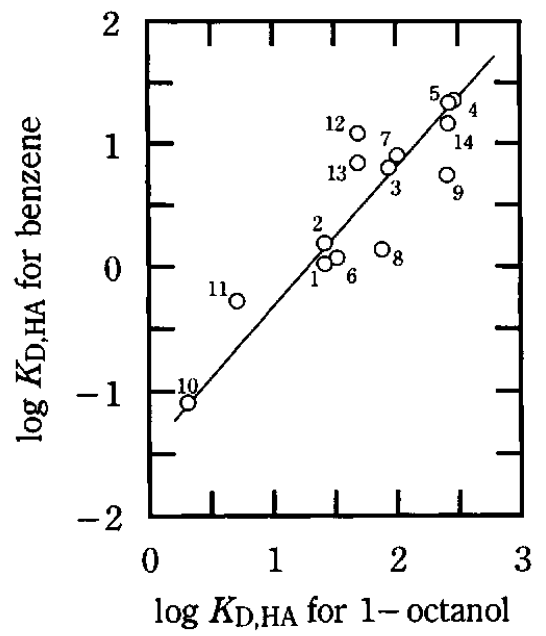

Fig. 5 Relationship of partition constant of carboxylic acids between the 1-octanol/water and benzene/water systems. Numbers are the same as in Fig. 3.

and decanoic acid by Fujii et al. ${ }^{18}$ This LFER can be expected to be established because their study was confined to a homologous series.

The correlation of the dimerization constants of the present acids with their $\mathrm{p} K_{\mathrm{a}}$ was not clearly recognized, but only within a homologous series of carboxylic acids the linear relationship between the dimerization constant and $\mathrm{p} K_{\mathrm{a}}$ of the acids was observed. The dimerization of cyclohexanecarboxylic, trans-2-butenoic, 2-hexenoic and 3-cyclohexene-1-carboxylic acids was found to be considerably inhibited, but that of benzoic and $\mathrm{m}$ methylbenzoic acids was found to be promoted.

A new relationship on the partition equilibrium of carboxylic acids between 1-octanol/water and benzene/water systems

Under the conditions when the dissociation of a carboxylic acid in the aqueous solution can be neglected, the partition equilibrium of the acid can be expressed as:

$$
(\mathrm{HA}) \rightleftharpoons(\mathrm{HA})_{\mathrm{o}} ; K_{\mathrm{D}, \mathrm{HA}}=[\mathrm{HA}]_{\mathrm{o}} /[\mathrm{HA}]
$$

for the 1-octanol/water system, and

$$
2(\mathrm{HA}) \rightleftharpoons(\mathrm{HA})_{2, \mathrm{o}} ; K_{2, \mathrm{HA}^{\prime}}=\left[(\mathrm{HA})_{2}\right]_{\mathrm{o}} /[\mathrm{HA}]^{2}
$$

for the benzene/water system, respectively, where $(\mathrm{HA})_{2,0}$ denotes the dimeric carboxylic acid in the benzene phase and the subscript " 0 " refers to the organic phase. The constant $K_{2, \mathrm{HA}^{\prime}}$ can be regarded as the overall dimerization constant of the carboxylic acid between the two phases, and can be expressed as:

$$
K_{2, \mathrm{HA}^{\prime}}=K_{2, \mathrm{HA}} \cdot K_{\mathrm{D}, \mathrm{HA}}{ }^{2} .
$$

These overall constants ( $K_{\mathrm{D}, \mathrm{HA}}$ for 1-octanol/water and $K_{2, \mathrm{HA}^{\prime}}$ for benzene/water) can be expected to be the measure of the affinity of carboxylic acid for the respec- 
Table 2 Partition and dimerization constants of aliphatic carboxylic acids

\begin{tabular}{|c|c|c|c|c|c|c|}
\hline \multirow{2}{*}{\multicolumn{2}{|c|}{ Acid }} & \multirow{2}{*}{$V_{\mathrm{HA}} / \mathrm{cm}^{3}$} & \multirow{2}{*}{$\mathrm{p} K_{\mathrm{a}}$} & \multirow{2}{*}{$\frac{1-\text { Octanol }}{\log K_{\mathrm{D}, \mathrm{HA}}}$} & \multicolumn{2}{|c|}{ Benzene } \\
\hline & & & & & $\log K_{\mathrm{D}, \mathrm{HA}}$ & $\log K_{2, \mathrm{HA}}$ \\
\hline 15 & Acetic & $57.2^{7}$ & $4.56^{15}$ & $(-0.12)$ & $-2.07^{17}$ & $2.16^{17}$ \\
\hline 16 & Propanoic & $74.6^{7}$ & $4.67^{15}$ & $(0.40)$ & $-1.36^{17}$ & $2.21^{17}$ \\
\hline 17 & Butanoic & $92.0^{7}$ & $4.63^{15}$ & 0.81 & $-0.79^{17}$ & $2.28^{17}$ \\
\hline 18 & Pentanoic & $108.8^{7}$ & $4.64^{15}$ & 1.36 & $-0.16^{17}$ & $2.36^{17}$ \\
\hline 19 & Hexanoic & $125.3^{7}$ & $4.84^{17}$ & $1.90^{19}$ & $0.31^{17}$ & $-2.45^{17}$ \\
\hline 20 & Heptanoic & $141.5^{7}$ & $4.89^{15}$ & 2.48 & $(0.90)$ & $(2.60)$ \\
\hline 21 & Octanoic & $158.7^{7}$ & $4.89^{15}$ & $(2.91)$ & (1.49) & (2.39) \\
\hline 22 & Nonanoic & $174.7^{7}$ & $4.89^{17}$ & (3.39) & $(2.08)$ & $(2.29)$ \\
\hline 23 & Decanoic & $194.5^{7}$ & $4.92^{18}$ & (3.98) & $2.70^{18}$ & $2.42^{18}$ \\
\hline
\end{tabular}

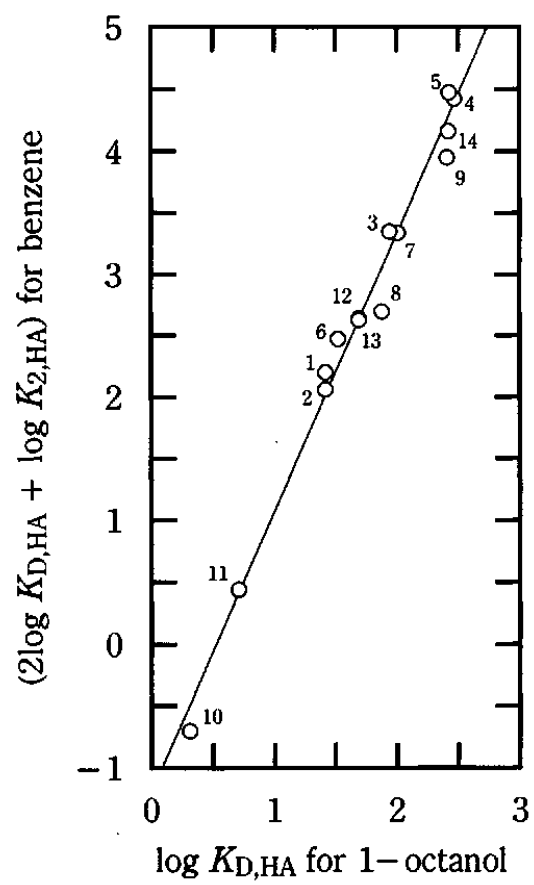

Fig. 6 Correlation of $\log K_{\mathrm{D}, \mathrm{HA}}$ for the 1-octanol/water system with $\left(2 \log K_{\mathrm{D}, \mathrm{HA}}+\log K_{2, \mathrm{HA}}\right)$ for the benzene/water system. The solid line is a straight line drawn by the least-squares method $(Y=2.26 X-1.20$ with a correlation coefficient, $R=0.99$ ). Numbers are the same as in Fig. 3.

tive organic phase. The plots of $\left(\log K_{2, \mathrm{HA}}+2 \log K_{\mathrm{D}, \mathrm{HA}}\right)$ for the benzene/water system against $\log K_{\mathrm{D}, \mathrm{HA}}$ for the 1octanol/water system are shown in Fig. 6. As shown in Fig. 6, the plots fit well with the straight line with a correlation coefficient, $R=0.99$ drawn by the leastsquares method, irrespective of the kinds of carboxylic acids, in contrast to the plots in Figs. 3-5. Then, the dimerization constant of a carboxylic acid in the benzene phase can be calculated making use of this new relationship and the additivity of the partition constant of the monomeric carboxylic acid. In addition, the present method is useful for the estimation of the partition and dimerization constants of carboxylic acids whose constants are difficult to be determined. The constants can be estimated as follows.

The partition and dimerization constants of heptanoic, octanoic and nonanoic acids, whose distribution ratios are difficult to be determined, calculated on the basis of the present method are designated in parentheses in Table 2. The values in parentheses were calculated by the following manner: First, the values of $\log K_{\mathrm{D}, \mathrm{HA}}$ for the 1-octanol/water system were estimated from the molar volumes of the respective acids according to the solid straight line shown in Fig. 4. Secondly, the values of $\left(2 \log K_{\mathrm{D}, \mathrm{HA}}+\log K_{2, \mathrm{HA}}\right)$ for the benzene/water system were estimated from the values of $\log K_{\mathrm{D}, \mathrm{HA}}$ for the 1octanol/water system on the basis of the relationship $\left(2 \log K_{\mathrm{D}, \mathrm{HA}}+\log K_{2, \mathrm{HA}}=2.26 \log K_{\mathrm{D}, \mathrm{HA}}-1.20\right)$ shown in Fig. 6. Subsequently, the values of $\log K_{\mathrm{D}, \mathrm{HA}}$ for the benzene/water system were calculated by adding 0.59 per one methylene moiety ${ }^{8}$ to the value of $\log K_{\mathrm{D}, \mathrm{HA}}$ of the parent carboxylic acid (hexanoic acid) whose partition constant is known, on the basis of the additivity of the partition constant. Then, the values of $\log K_{2, \mathrm{HA}}$ were calculated by subtracting the values of $2 \log K_{\mathrm{D} \text {, HA }}$ for the benzene/water system from the values of $\left(\log K_{2, \mathrm{HA}}+2 \log K_{\mathrm{D}, \mathrm{HA}}\right)$ of the respective carboxylic acids.

The present new relationship can be expected to be established for the other inert solvents/water systems similar to the benzene/water system. Further, this relationship can be applied also to the partition equilibrium of alkylphosphoric acids which are known to be dimerized in inert solvents as well as carboxylic acids.

\section{References}

1. T. Fujita, J. Iwasa and C. Hansch, J. Amer. Chem. Soc., 86, 5175 (1964).

2. C. Hansch and S. M. Anderson, J. Org. Chem., 32, 2583 (1967).

3. C. Hansch, J. E. Quinlan and G. L. Lawrence, J. Org. Chem., 33, 347 (1968).

4. A. Leo, C. Hansch and D. Elkins, Chem. Rev., 71, 525 (1971).

5. C. Hansch and A. Leo, "Substituent Constants for Correlation Analysis in Chemistry and Biology", John Wiley 
\& Sons, New York, 1979.

6. H. Yamada, K. Yajima, H. Wada and G. Nakagawa, Talanta, 42, 789 (1995).

7. David R. Lide, "CRC Handbook of Chemistry and Physics", 74th ed., 1993 - 1994, CRC Press, Boca Raton, 1993.

8. Y. Fujii and M. Tanaka, Bull. Chem. Soc. Jpn., 54, 3696 (1981).

9. "Beilsteins Handbuch der Organischen Chemie", Beilstein Institute, Springer-Verlag, Berline, 1970, 1976.

10. H. Yamada and C. Kato, Talanta, 40, 1049 (1993).

11. H. Yamada, Y. Taguchi and H. Wada, Talanta, 41, 573 (1994).

12. H. Yamada, M. Ito and M. Mizuta, Bull. Chem. Soc. Jpn., 60, 3557 (1987).

13. H. Yamada, K. Adachi, Y. Fujii and M. Mizuta, Solvent Extr. Ion Exch., 4, 1109 (1986).
14. H. Yamada, S. Horikawa, Y. Fujii and M. Mizuta, Bull. Chem. Soc. Jpn., 61, 835 (1988).

15. E. Martell and R. M. Smith, "Critical Stability Constants", Vol. 3, Plenum Press, New York, 1977.

16. E. P. Serjent and B. Dempsey, "Ionization Constants of Organic Acids in Aqueous Solution", p. 171, Pergamon Press, Oxford, 1979.

17. I. Kojima, M. Yoshida and M. Tanaka, J. Inorg. Nucl. Chem., 32, 987 (1970).

18. Y. Fujii, Y. Kawachi and M. Tanaka, J. Chem. Soc., Faraday Trans. 1, 77, 63 (1981).

19. H. Yamada, C. Kato and M. Mizuta, Bull. Chem. Soc. Jpn., 65, 186 (1992).

(Received May 26, 1997)

(Accepted September 8, 1997) 